\title{
CORRIGENDUM
}

\section{Dual E1A oncolytic adenovirus: targeting tumor heterogeneity with two independent cancer-specific promoter elements, DF3/MUC1 and hTERT}

\author{
JC Doloff, Y Jounaidi and DJ Waxman
}

Cancer Gene Therapy (2011) 18, 228; doi:10.1038/cgt.2011.1

Correction to: Cancer Gene Therapy (2011) 18, 153-166; doi: 10.1038/cgt.2010.52; published online 24 September 2010

The publication by Doloff et al. is revised to include Y Jounaidi as a co-author. The eighth sentence under the subheading 'Construction of Adeno-DF3-E1A/hTERTE1A' should read 'The entire ICeuI-PISceI pShuttle fragment containing the DF3-E1A and hTERT-E1A expression cassettes was then ligated into the adenoviral genome using the Adeno-X expression system, as per the manufacturer's instructions (Clontech Labs, Mountain View, CA).' In addition, the Acknowledgments should read 'The authors thank Michael Durando for his assistance in generating the holoclones. Supported in part by NIH grant CA49248 (to DJW) and by Susan G Komen Breast Cancer Foundation grant BCTR0504032 (to YJ).' 